\title{
Islam in deutschen Medien
}

\author{
Sabine Schiffer
}

Wenn es um „Islam“ in deutschen Medien gehen soll, könnten wir diesen Aufsatz schnell beenden. Der Islam als Religion und eigentliches Thema kommt in den Massenmedien kaum vor und fast gar nicht das religiöse Selbstverständnis von Muslimen. ${ }^{1}$ Kommen Muslime selbst zu Wort, dann oft als Befragte zu Themen, die andere implementiert haben. Das Islambild der Medien ist ein eigenes Konstrukt und bildet damit keine Ausnahme. Denn immer dann, wenn uns ein direkter Zugang zu einem Thema, Land, Menschen fehlt, dominieren die Wahrnehmungsstrukturen, die uns eine problemzentrierte Berichterstattung vorgibt. ${ }^{2}$ Wenn sich deren Folgen dann noch in medialen Folgeprodukten wie Film oder Talk niederschlagen, ergibt sich ein Wiederholungseffekt mit starker Überzeugungskraft und unabhängig vom Wahrheitsgehalt des Dargestellten.

In der Talkshow „Menschen bei Maischberger“ zum „Beschneidungsstreit“, der Juden und Muslime gleichermaßen betrifft, gab es unter anderem einen kurzen Einspieler über Rechtsfragen rund um Islam und Muslime. ${ }^{3}$ Es ging um die Kopftuchverbote in acht Bundesländern für Lehrerinnen, um das Schächten und die Möglichkeit einer Rechtsprechung nach islamischem Zivilrecht - der Scharia - in Deutschland sowie um das Urteil an einem Frankfurter Gericht, das einem muslimischen Ehemann ein Züchtigungsrecht für seine Frau zusprach. Die in wenigen Minuten erreichte, verkürzte bis fehlerhafte Reproduktion medialer Aufgeregtheiten ist nicht neu. En passant wird ein Islambild geformt oder

\footnotetext{
${ }^{1}$ Eine Ausnahme bildet hier das „Islamische Wort“ des SWR, das aber nur wenigen bekannt sein dürfte, unter: http://www.swr.de/islamisches-wort (31.08.2012). Natürlich ließe sich der Medienbegriff auch auf Schulbücher anwenden, die vom Georg Eckert Institut ausführlich untersucht wurden, dies wird aber im Rahmen dieses Beitrags nicht möglich sein. Darum sei an dieser Stelle auf die interessanten Forschungsergebnisse verwiesen: http:/www.gei.de/aktuelles/mitteilungen/mitteilung-details/article/ modernes-europa-versus-antiquierter-islam-die-darstellung-von-islam-und-muslimen-in.html (31.08.2012). Zur Selbstdarstellung des Islam in Schulbüchern für Islamischen Religionsunterricht und Islamkunde vgl. Klaus Spenlen und Susanne Kröhnert-Othman (Hrsg.) 2012.

${ }^{2}$ Vgl. Sabine Schiffer 2005.

${ }^{3}$ ARD, 14. August 2012: 22.45 Uhr.
} 
bestätigt, das viel mit Vorurteilen und medialen Produktionsprozessen und wenig mit dem Objekt der Betrachtung zu tun hat.

Die Einbeziehung fremden Zivilrechts in die hiesige Rechtsordnung ist ein deutsches Rechtsprinzip und wird seit Jahrzehnten auf das Zivilrecht sowohl sog. islamischer als auch anderer Staaten angewandt, sofern es die Betroffenen wünschen. Denn die Beteiligten können sich auch nach deutschem Zivilrecht aburteilen lassen, wenn es für sie vorteilhafter ist. Genau dies strebte eine deutsche Konvertitin an, als sie sich vorzeitig von ihrem marokkanischen Ehemann wegen dessen Gewalttätigkeit scheiden lassen wollte. Diesem Wunsch gab eine Frankfurter Richterin nicht statt, weil diese dem Muslim ein Züchtigungsrecht zusprach. Ihr rassistisches Urteil, das die Ehefrau weiterhin einem Martyrium ausgeliefert hätte, wurde auf juristischer Ebene sofort korrigiert. Die Richterin wurde vom Dienst suspendiert, ein Kollege sprach der Klägerin das vorzeitige Trennungs- und Scheidungsrecht zu. Dieses Faktum verhinderte jedoch weder die Darstellung in der Springer-Zeitung „Die Welt“, auf die sich Maischberger bezieht, noch den Spiegel-Titel „Mekka Deutschland“ vom 26. März 2007. Letzterer behauptete, Muslime würden in Deutschland ihr eigenes Rechtssystem durchdrücken und das ganze Land „islamisieren“. Und genau dieses Angstszenario reproduziert Sandra Maischberger in ihrer Sendung und stellt die falsch schlussfolgernden Fragen: „Ist das zu viel Religionsfreiheit?“ und „Wie viele Ausnahmeregelungen [sic!] verträgt denn ein Staatswesen?"

Außer beim Schächten handelt es sich jedoch nicht um Ausnahmeregelungen: Weder sind wir ein laizistischer Staat, der religiöse Symbolik wie das Kopftuch aus öffentlichen Räumen verbannen müsste, noch handelt es sich bei der Rechtsprechung und auch nicht beim Schiedsmannswesen um „Ausnahmen“, denn alles ist nach deutschem Recht geordnet und kann nur dann Anwendung finden, wenn es nicht grundgesetzlichen Bestimmungen widerspricht. ${ }^{4}$

Das Beobachtete ist symptomatisch auch in Zeiten, in denen viele Medienmacher glauben, sie hätten ein Problembewusstsein für das entwickelt, was Studien in den letzten Jahren vermehrt bemängeln: Medien tragen zu einer antiislamischen Stimmung bei. Die gut belegte Studie vom Institut für Kommunikationswissenschaft der Universität Jena, die Wolfgang Frindte und Nicole Haußecker

\footnotetext{
${ }^{4}$ Vgl. Mathias Rohe ${ }^{2} 2001$.
} 
unter dem Titel „Inszenierter Terrorismus“ 2010 herausgaben, bezeugt dies exemplarisch. Sie zeigt unter anderem, dass sich unkritisch kolportierte Terrorwarnungen beim Publikum nicht primär als Angst vor Terror niederschlagen, sondern als Angst vor dem Islam.

Wenig Bewusstsein scheint von Medienseite nach wie vor für Fragen des Agenda-Settings zu bestehen, etwa wenn man sich zu einer Art Verlautbarungsjournalismus für Lobbyorganisationen oder Ministerien hinreißen lässt. So folgt das Agenda-Setting um Islamismus und Salafismus stark der Veröffentlichungspraxis von Pressemitteilungen des Bundesministeriums des Innern (BMI): Während es zur Razzia gegen Salafisten im Juni 2012 eine entsprechende Pressemitteilung gab, fehlte eine solche in Bezug auf die verfassungsrechtlich sehr relevante Zusammenkunft von Vertretern des Bundeskriminalamts, der Bundespolizei und der Verfassungsschutzämter ungefähr zur gleichen Zeit, die lediglich auf der Website des BMI Erwähnung fand. ${ }^{5}$ Ersteres wurde zum breiten Medienthema, Letzteres nicht. Auch die Veröffentlichungspraxis bezüglich einer weiteren Studie von Prof. Frindte selbst gab Anlass zur Kritik und einer Stellungnahme des Studienleiters, die vonseiten großer Tageszeitungen auch aufgegriffen wurde. Zu dem Zeitpunkt war jedoch die alarmistische Botschaft des Innenministers über ,integrationsunwillige Muslime“ schon verlautbart vor allem in den Nachrichtenformaten des Leitmediums Fernsehen. ${ }^{6}$

Die genannten Beispiele sind so singulär wie symptomatisch. Eine Aufarbeitung ist angesichts der Fülle von Nachrichten kaum mehr möglich und trägt immer den Ruch des Relativierens in sich, angesichts dominanter antiislamischer Einstellungen. Darum ist es sinnvoll, sich mit einigen grundlegenden Mechanismen der Konstruktion eines Bildes vom Anderen auseinander zu setzen - in dem Fall am Beispiel von Islam und Muslimen.

\footnotetext{
${ }^{5}$ Vgl. Sabine Schiffer 2012.

${ }^{6}$ Vgl. bspw. die Berichterstattung der Süddeutschen Zeitung mit der der Tagesschau Anfang März 2012 .
} 


\section{Das Framing des Islams: \\ Frames und ihre nachhaltige Wirkung}

Unser Islambild wurde im Wesentlichen durch die Auslandsberichterstattung geprägt, mindestens seit der Iranischen Revolution 1979. ${ }^{7}$ Starke, bis heute wirksame Rahmen der Wahrnehmung - Frames - sind entstanden. In diese Schablonen werden neuere Entwicklungen schnell eingepasst. So ist heute noch der Frame der Frauenunterdrückung dominant, den Betty Mahmoody mit ihrem Buch und Film „Nicht ohne meine Tochter“ gesetzt hat. Dies mag eine mögliche Erklärung dafür sein, warum beispielsweise Ayaan Hirsi Magan alias Hirsi Ali mit dem Axel-Springer Ehrenpreis ausgezeichnet wurde und auch weiterhin eine hohe Glaubwürdigkeit genießt, obwohl sie über ihre Herkunft, ihre angebliche Verfolgung und ihre Ehe nachweislich gelogen hat. ${ }^{8}$ Ihre indirekte Rechtfertigung der Untaten Anders Breiviks in ihrer Ehrenpreisrede erregte nur wenig Aufsehen. ${ }^{9}$ Sie passt einfach in den Frame der Frauenunterdrückung als eine Frau, die sich aus den Fängen „des Islams" befreit habe. Auch der Frame der Gewaltaffinität von Muslimen oder einer Feindschaft gegenüber Kunst- und Meinungsfreiheit im Zusammenhang mit der Affäre um Salman Rushdies Buch „Die satanischen Verse" 1989 ist virulent. Während diese Interpretation in Bezug auf den Mordaufruf Ayatollah Khomeinis gegenüber Rushdie stimmte, wurde die gleiche Interpretationsschablone voreilig auf den Karikaturenstreit angewandt, wo es weniger um Kunstfreiheit ging, sondern vielmehr um eine gezielte und bösartige Provokation. ${ }^{10}$

Wie schwer man sich damit tut, etablierte Frames zu überwinden, ließ sich beim Umgang mit dem Mord an Marwa El Sherbiny ersehen - Muslime als Opfer schienen nicht vorstellbar, so dass Politik und Medien lange brauchten, bis sie die Islam feindliche Dimension der Tat erkannten. ${ }^{11}$ Inwiefern die neuen

\footnotetext{
${ }^{7}$ Vgl. Kai Hafez 2002.

${ }^{8}$ siehe „Ayaan Hirsi Ali Lies Exposed“ in dailymotion.com, unter: http://www.dailymotion.com/video/ xa09uv_ayaan-hirsi-ali-lies-exposed-part-1_people 1-4 (31.08.2012).

${ }^{9}$ Details zur Rede und dem Medienecho finden sich zusammengefasst in: Schiffer; Sabine „Ayaans Ruf nach Mord“ in migazin.de, 29.05.2012, unter: http://www.migazin.de/2012/05/29/ayaans-ruf-nach-mo rd-blieb-nicht-ganz-ungehort/ (31.08.2012).

${ }^{10} \mathrm{Vgl}$. Xenia Gleißner und Sabine Schiffer 2008.

${ }^{11}$ Der sehr lesenswerte Beitrag von Stephan Kramer „Im Zeichen der Solidarität mit allen Mus-
} 
Bilder aus Nahost vom sog. arabischen Frühling unsere Vorurteile zu korrigieren vermögen, bleibt abzuwarten. Die religiösen Frames zur Interpretation der aktuellen Entwicklungen stehen schon bereit, wie nicht zuletzt ein Focus-Titel vom 7. Februar 2011 nahe legt: „Kann der Islam [sic!] Freiheit?“ heißt es da framekonform auf der Titelseite. Die alten Rahmen der Wahrnehmung, deren Filterfunktion und der Zuweisung bestimmter Problematiken auf „den Islam“ könnten sich langfristig als dominant erweisen, weil sie schon so lange etabliert sind. Und dies birgt die Gefahr, die vielen anderen und möglicherweise relevanteren Aspekte vor Ort zu übersehen. ${ }^{12}$

So wird seit Jahren gar keine Islamdebatte geführt, sondern eine Islambilddebatte, die mehr über die Bildträger aussagt, als über das Objekt der Betrachtung. Die Bildträger wählen ja die zu betrachtenden Aspekte aus - nach ihren Erwartungen. Und so bestimmen die Vorurteile das, was auch zukünftig bemerkt werden kann. Dabei gilt: Alles, was wir wissen, ist (weitestgehend) wahr. Es ist eben nur nicht alles. Und es ist so extrem wenig von dem vielfältigen Ganzen, dass ein völlig verzerrtes Bild entstanden ist - aber ein wirkmächtiges, weil es schon so oft wiederholt wurde. Und Wiederholung hat die stärkste Überzeugungskraft schlechthin. ${ }^{13}$

\section{Zur Konstruktion des Islambilds}

Das Bild ist entstanden aus bestimmten Fakten, die ausgewählt und in den Vordergrund gerückt werden. Es sind mediale Konstruktionen. Medienverantwortliche bestimmen, welche Aspekte gezeigt und welche ausgeblendet werden, welche vergrößert und welche verkleinert werden. Vergleicht man die EuropolStatistiken zum Terrorismus mit den lupenartigen Vergrößerungen einzelner

\footnotetext{
limen" vom 13.07.2009 auf www.qantara.de macht auf diese Problematik aufmerksam, unter: http: //de.qantara.de/Im-Zeichen-der-Solidaritaet-mit-allen-Muslimen/2671c2765i1p127/index.html (31.08.2012).

12 Interview mit der Autorin in: "Die Ökonomie entscheidet" (taz 31.07.2011).

${ }^{13}$ Z. B. Marian Schwartz: „Repetition and rated truth value of statements,” In: Journal of Psychology vol. 95/Nr. 3, 1982: S. 393-407, unter: http://www.jstor.org/pss/1422132 (31.08.2012); William James sagte: „Nichts ist zu absurd, dass es nicht geglaubt würde, wenn es nur oft genug wiederholt worden ist!“ zitiert nach: Ozubko, Jason und Fuelsang, Jonathan: „Remembering makes evidence compelling: Retrieval from Memory can Give Rise to the Illusion of Truth," In: Journal of Experimental Psychology; Vorabveröffentlichung online 8.11.2010, unter: http://www.reason.uwaterloo.ca/Site/OzubkoFugelsang_11.pdf (31.08.2012).
} 
Terroranschläge mit muslimischen Akteuren in den Medien und den entsprechenden politischen Debatten, dann kann man das Potential der Verzerrung abschätzen. ${ }^{14}$ Während etwa die große Anzahl von ETA-Anschlägen als Gefahr für deutsche Touristen medial kaum eine Rolle spielt, ist sie auch weniger im Bewusstsein. Anders bei Anschlägen oder Anschlagsversuchen aus dem sog. islamistischen Spektrum. Kein Thema sollte jedoch erörtert werden, ohne dessen mediale Konstruiertheit zu erfassen. Schließlich können ganze Talkshow-Zyklen die relevanten Zusammenhänge verfehlen.

$\mathrm{Zu}$ analysieren sind als Teil der Inhaltsanalyse bzw. über die reine Inhaltsanalyse hinausgehend immer noch mindestens

- die Nominationspraxis,

- eine Markierungspraxis mitsamt daraus resultierender Prämissen,

- faktizierende und relativierende Darstellungselemente,

- komplexe Metaphern und deren implizite und idealisierte Maßstabssetzung,

- Platzierungs- und Raumfragen mit Blick auf die Aufmerksamkeitssteuerung.

\subsection{Die Nominationspraxis}

Je nach ausgewählter Benennung erscheint der zu beschreibende Sachverhalt in einer bestimmten Perspektive. Bezeichnet man einen Attentäter als „Amokläufer“ oder „Terrorist“, als „Fanatiker“, „Rebell“ oder „Freiheitskämpfer“, so wird der Tat implizit mehr oder weniger Legitimation zugesprochen. Darum sind die sich durchsetzenden Benennungspraxen jeweils zu untersuchen. Und natürlich erhält die Nichtübersetzung des arabischen Wortes für Gott, „Allah“, den Eindruck von Fremdheit und Polytheismus aufrecht - egal, ob dieser Fehler von Muslimen oder Nichtmuslimen begangen wird. Aus journalistischer Perspektive ist es jedoch wichtig, sich selbst zu prüfen, wann man sich nämlich auf Selbstaussagen von Muslimen beruft und wann man diese als irrelevant ablehnt. Die Diskurs- und Medienanalyse hilft, die Inkohärenzen in der Bewertung aufzuspüren.

\footnotetext{
${ }^{14}$ Die Anschläge der ETA würden die Nachrichtenwertfaktoren erfüllen - als Gefahr etwa für deutsche Touristen in Spanien -, spielen medial aber allenfalls eine untergeordnete Rolle.
} 


\subsection{Eine Markierungspraxis mitsamt daraus resultierender Prämissen}

Die Nominationsanalyse berührt bereits den viel komplizierteren Komplex der Präsuppositions- bzw. Prämissenanalyse. Prämissen erweisen sich ob ihrer Subtilität als besonders stark und resistent gegenüber Aufklärung, weil sie nur schwer explizit angegriffen werden können. Die Frage „Wie gefährlich ist der Islam?" setzt bereits eine Gefährlichkeit voraus, da lediglich nach dem Grad gefragt wird. ${ }^{15}$ Vergleichbare Unterstellungen sind auch durch Sinn-Induktionsphänomene zu erreichen, etwa indem man entsprechende Fotos, Begriffe oder Symbole in einem Kontext verwendet, der problematisch ist bzw. nicht spezifisch oder gar relevant für den Sachverhalt oder die fokussierte Gruppe. Etwa der Hinweis auf die Durchführung einer Genitalverstümmelung an Mädchen „in der Nähe einer Moschee“ ist irrelevant, da diese altafrikanische Praxis in muslimischen, christlichen und animistischen Communities durchgeführt wird. ${ }^{16}$ Durch die räumliche Nähe im Ko(n)text, die auch durch Adjektivierung oder das Einstreuen von Nebensätzen zu erreichen ist, werden Zusammenhänge unterstellt, die zu prüfen wären. Etwa bei der Polemik des streitbaren konservativen Predigers Qaradawi gegen die Weihnachtsdekoration 2009 in Doha, der Hauptstadt Katars: Hier kann die Kontextlosigkeit nur zu dem Missverständnis führen, der Islamist würde gänzlich Weihnachten ablehnen.

\subsection{Faktizierende und relativierende Darstellungselemente}

Als faktizierendes Element erweisen sich nicht nur bestimmte Artikel (Definitpartikel), der verwendete Modus, sondern auch die Verwendung von Aktiv- oder Passivkonstruktionen - wie natürlich wiederum entsprechende Nominationen, ob etwa eine Aussage mit dem Verb „versichert“, „sagt“ oder „behauptet“ oder eben mit „wurde behauptet“ präsentiert wird. Die Verwendung von bewertenden Partikeln wie „ehrlicherweise“ oder „paradoxerweise“ markieren zwei gegensätzliche Pole der Skala, um Vorkommnisse als erwartbar und damit wahr oder eben als unwahr zu markieren, wobei gerade Letzteres ein effektives Mittel ist, um Abweichungen vom eigenen Bild als Ausnahme nahe zu legen.

\footnotetext{
15 Titelseite Stern 13.09.2007.

${ }^{16}$ Vgl. Anika Rahman und Nahid Toubia 2000.
} 


\subsection{Komplexe Metaphern und deren implizite und idealisierte Maßstabssetzung}

Eine dominante komplexe Metapher im Kontext der Abwertung von Islam und Muslimen ist die der Emanzipation als Fortschrittsgradmesser. Selbstidealisierend wird auf die angeblich realisierte "Gleichstellung der Frau“ [sic!] hingewiesen und auf die Bekämpfung der Homosexuellendiskriminierung. Auch hier könnte man von einem Frame sprechen, der als Maßstab benutzt wird, um eine andere Kultur abzuwerten - denn auch hier gilt, dass die Auswahl von Aspekten die Vergleichsergebnisse bestimmt und eher Stereotype reproduziert, statt sie korrigiert. In Bezug auf Islam und Muslime werden vielfach entmenschlichende Metaphern angewandt, die entweder Krankheit (Irrsinn, Krebsgeschwür) oder Gefahr (Bombenallusionen) heraufbeschwören. ${ }^{17}$

\subsection{Platzierungs- und Raumfragen mit Blick auf die Aufmerksamkeitssteuerung}

Unsere repräsentative Analyse im Rahmen des Projektes Islam-Media-Watch aus dem Jahre 2003 hat ergeben, dass die aufmerksamkeitsrelevant platzierten Beiträge zum Themenfeld „Islam“ vor allem problemzentriert waren, während differenzierte Beiträge in Print und Fernsehen vorhanden waren, aber generell nicht gut platziert wurden und somit weniger wahrgenommen werden konnten. Diesbezüglich wäre eine aktuelle Analyse erforderlich, um zu prüfen, inwiefern die vernehmbaren guten Vorsätze zur Forderung nach mehr Ausgewogenheit von Medienmacherseite umgesetzt werden.

Die einmal etablierte Konstruktion eines Bildes ist aber auch darum so wirkmächtig, weil unsere Sprache uns oftmals einen Streich spielt. ${ }^{18}$ Nicht nur, dass unser Unterbewusstsein Verneinung nicht erkennt und jede Ablehnung eines Stereotyps als Wiederholung empfindet, auch die positive Betonung von Selbstverständlichem impliziert genau dort eine Problematik, wo man keine vermutet wissen will. ${ }^{19}$ Etwa „Der Islam ist mit unseren Werten vereinbar!“ ist ein solch

\footnotetext{
${ }^{17}$ Vgl. Jürgen Link 2007 mit Westergaard-Bombenkopf.

${ }^{18}$ Vgl. Sabine Schiffer 2010a.

${ }^{19}$ Vgl. Christoph Butterwegge und Gudrun Hentges 2006.
} 
kontraproduktiver Satz, der das „Wir vs. Ihr“ betont und eine Vereinbarkeitsproblematik präsupponiert.

Zur Problematik der Sprach- und Bildproduktion sowie deren Kombination gesellt sich noch die Rezeption durch das Medienpublikum, das einen nicht unerheblichen Teil der Verantwortung trägt für das, was es aus den Darstellungsweisen schließt. Ein verallgemeinernder Reflex geschieht - neben der Wirkung von Wahrnehmungsfiltern durch die eigene Erwartung - vor allem aus einer Außenperspektive heraus, während aus einer Innenperspektive heraus berichtete Skandale eher als Abweichung von der Norm interpretiert werden: Dies geschah etwa bei den Missbrauchsskandalen in christlichen Einrichtungen hierzulande. Während Empörung und Ablehnung groß und gut zu vernehmen waren, hätte Vergleichbares in muslimischen oder anderen Ländern sehr vermutlich nicht zu den gleichen Reflexen mit einer Solidarität den empörten Muslimen gegenüber geführt, sondern viel wahrscheinlicher zu einer verallgemeinerten ablehnenden Haltung gegenüber der Out-Group nach dem Motto: „Aha, bei denen ist das also so!" Umgekehrt geschah dies auch, als ich im Ausland gefragt wurde, warum wir in Deutschland unsere Kinder umbrächten.

Ein verallgemeinerndes Stereotyp machen erst die Betrachter aus den berichteten Vorfällen, wenn sie bestimmte Vorkommnisse als Eigenschaften - etwa neuerdings von sog. Salafisten - pars-pro-toto einer ganzen Gruppe zuweisen, in dem Fall der großen, heterogenen Gruppe der Muslime. Der beliebte Einsatz kollektiver Symboliken durch Bildmedien wirkt da wie ein Katalysator.

Was Sprachfallen und Diskursschleifen anbelangt, bietet die Analyse des antisemitischen sowie des anti-antisemitischen Diskurses des ausgehenden 19. Jahrhunderts ein erhellendes Lernfeld. ${ }^{20}$ Für die Nutzbarmachung der Ergebnisse der Antisemitismusforschung für die allgemeine Vorurteilsforschung plädiert nicht zuletzt der ehemalige Leiter des Zentrums für Antisemitismusforschung Wolfgang Benz. ${ }^{21}$ Die Erkenntnisse aus diesem Forschungszweig machen deutlich, dass die Bekämpfung von Antisemitismus nicht die Juden oder das Judentum in den Fokus nimmt, sondern die Träger des Ressentiments und deren (bewusste oder unbewusste) Konstruktionen.

\footnotetext{
${ }^{20}$ Vgl. Sabine Schiffer und Constantin Wagner 2009.

${ }^{21}$ Vgl. Wolfgang Benz 2009, 2010.
} 
Eine Folge der Konstruktion des Fremdbilds Islam ist die Übertragung auf die jeweiligen Muslime, die in europäischen Ländern leben. So wurde der Diskurs über „den Islam“ zu einem Teil des „guten alten“ Ausländerdiskurses. Stark verkürzt ließe sich sagen: Aus dem Italiener ist ein Türke und aus dem Türken ist ein Muslim geworden im Laufe der jahrzehntelangen Betrachtung, die übrigens immer dann vermehrt einsetzte, wenn es konjunkturelle Schwierigkeiten gab. Heute wird nicht selten eine Statistik oder ein Bericht über Einwanderung oder Integration mit dem Bild einer kopftuchtragenden Frau geschmückt. ${ }^{22}$

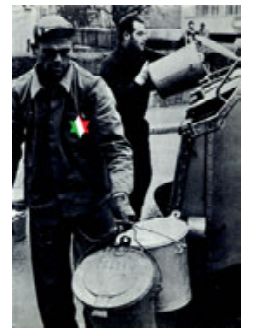

Foto aus den 1970ern

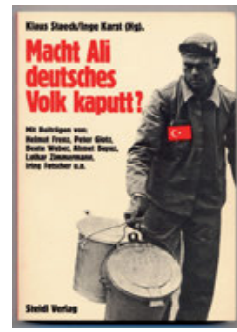

1974

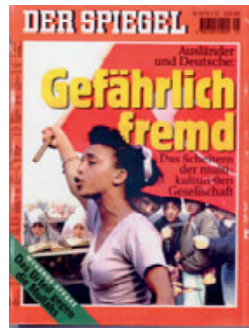

1997

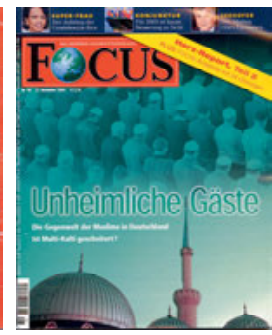

2004

Nun könnte man einerseits aus dem Diskurs über die Italiener lernen, wie man - ohne dass „Integrationsleistungen“ wie etwa ein besonderer Bildungserfolg erbracht wurden - eine enorme Imageverbesserung erreicht hat. Auf der anderen Seite scheint die vermehrte Markierung von tatsächlich oder vermeintlich Islamischem zu einer Verschiebung der Wahrnehmung von der Nationalität hin zur Religion geführt zu haben. Aus den Türken der 1980er und 1990er Jahre erinnert sei an die Anschläge in Mölln und Solingen - sind inzwischen Muslime geworden.

\section{Wenn die Analyse nicht stimmt, verfehlen auch die Lösungsansätze}

Der Diskurs über Islam und Muslime kann als Defizitdiskurs bezeichnet werden, weil er primär Defizite aktualisiert und nicht von der Gleichwertigkeit der Kulturen ausgeht, die alle ihre Stärken und Schwächen haben. Die Debatte um Thilo Sarrazins Buch „Deutschland schafft sich ab“ befeuerte diesen defizitorientierten

\footnotetext{
${ }^{22}$ Vgl. Yasemin Shooman 2011, S. 59-76.
} 
Diskurs aufs Neue, der seit nur mehr 30 Jahren vorherrscht und sich manchmal noch althergebrachter Mythen aus den Hochzeiten des Orientalismus bedient. ${ }^{23}$ Die Anschläge vom 11. September 2001 haben - entgegen vielfacher Einschätzung - keine neue Qualität in die Debatte gebracht, allenfalls einen Quantitätsund Explizitheitsschub in Richtung „Feindbild Islam“ befördert. ${ }^{24}$

Entgegen der medialen Dominantsetzung und verbreiteten Einschätzung eines Versagens von muslimischer Seite belegen die sogenannten Heitmeyer Studien „Deutsche Zustände“, dass heute das antiislamische Ressentiment mit dem wachsenden Erfolg der Gruppe wächst. ${ }^{25}$ In Zeiten von Wirtschaftskrise und Abstiegsängsten macht sich eine gruppenbezogene Ablehnung gerade im gebildeten Mittelstand breit und trifft besonders die aufstiegsorientierten Muslime.

Darum wären unsere Medien etwas spät dran, wenn sie genau jetzt - nach langen Jahren vergeblichen Forderns von wissenschaftlicher Seite - auch oder gar betont die gelungenen Beispiele von Integration und Erfolg aus dem islamischen Bereich zeigten. Dies könnte das Ressentiment gerade noch verstärken. Wie Angelika Königseder vom Zentrum für Antisemitismusforschung zugespitzt formulierte: „Nicht die Putzfrau mit Kopftuch hat uns gestört, sondern die kopftuchtragende Akademikerin!"26

Denkt man Medien als Vierte Gewalt, als Kontrollinstanz der Machtinstanzen, dann wäre es ihre genuine Aufgabe, kritisch auf derlei Zusammenhänge hinweisen. Dies gelingt auch immer wieder: etwa wenn SWR und Süddeutsche Zeitung aufdecken, dass im Münchner Prozess gegen die „Globale Islamische Medienfront" (GIMF) die wichtigste Person nicht vor Gericht steht, nämlich der Kopf der Gruppe, der Kontakte zum Verfassungsschutz hatte. ${ }^{27}$ Häufig jedoch dominiert der Eindruck, dass die großen Medien mehrheitlich die Debatten um die Minderheit in der Minderheit - nämlich sog. Integrationsverlierer, Menschen mit radikalen Ansichten oder auch Verbrecher unter Muslimen - geradezu befeuern (s. o. Maischberger z. B.). Hierin könnte einer der Gründe für den Erfolg

\footnotetext{
${ }^{23}$ Vgl. Edward Said ${ }^{2}$ 1997, Iman Attia 2007.

${ }^{24}$ Vgl. Sabine Schiffer 2005.

${ }^{25}$ Vgl. Wilhelm Heitmeyer 2010.

${ }^{26}$ Angelika Königseder 2009, S. $21 \mathrm{ff}$.

${ }^{27}$ Vgl. Holger Schmidt 2011-2012, unter: http://www.swr.de/blog/terrorismus/category/gimf/ (31.08.2012).
} 
des Internets als Alternative zum empfundenen Mainstream liegen, welches allerdings anderen Problemen unterliegt und sich in Sachen Rassismuskritik noch nicht besonders hervorgetan hat.

Die Analyse muss stimmen, um die richtigen Maßnahmen ergreifen zu können. Es mag durch die Aufmerksamkeitsverschiebung der letzten Jahrzehnte von der nationalen zur religiösen Kategorie durchaus auch Interesse am Islam an sich entstanden sein: Wissen über den Islam als solches ist aber nur bedingt notwendig, um einem antimuslimischen Rassismus zu begegnen. Das aufkommende Feindbild China wird auch nicht durch Informationen über die Eigenheiten chinesischer Kultur korrigiert werden. Die voraussehbar in Zukunft kritisierten Eigenschaften Chinas, die jetzt auch schon alle da sind, würden durch ein Eingehen darauf als relevant in den Aufmerksamkeitskegel gerückt. Man sollte also vielmehr die Intentionen hinter dieser Fokussierung ernst nehmen und durchschauen.

Genauso hier: Die Funktion des antimuslimischen Rassismus im heutigen Diskurs gilt es zu ermitteln. Folgt man dem Blick des Rassisten auf das Objekt seiner Betrachtung und diskutiert über dessen tatsächliche oder imaginierte Eigenschaften, haben Rassist und Rassismus gesiegt. Es gilt also, genau die Kontexte zu prüfen, um die es jeweils geht. Wenn wir dem Blick von Rassisten auf die Eigenschaften ihrer Objekte der Betrachtung folgen, die momentan zufällig religiös markiert sind, arbeiten wir einer rassistischen Zuschreibung zu. ${ }^{28} \mathrm{Um}$ es nochmal mit einer Gegenprobe zu verdeutlichen: Ja, es gibt schwarze Vergewaltiger. Angesichts des Vorkommens von Vergewaltigungen weltweit sollte eine solche Feststellung als irrelevant erkannt werden und nicht dazu verleiten, über Besonderheiten von Schwarzen zu diskutieren. Auch hier ist noch viel Spielraum für Selbstreflexion - bei allen Beteiligten, aber vor allem bei denen, die die Themensetzungen (Agenda-Setting) des Diskurses bestimmen.

Aus dem Geschriebenen ergeben sich folgende Überlegungen bzw. Anregungen:

- Interreligiöser Dialog gehört dahin, wo die Menschen religiös interessiert sind. Im unglücklichsten Fall könnte er - als politisches Instrument miss-

\footnotetext{
${ }^{28}$ Vgl. Sabine Schiffer und Constantin Wagner 2009.
} 
braucht - der Religionisierung anders gearteter Debatten noch weiter zuarbeiten.

- Wissen über islamische Geschichte als Vorläufer unserer Wissenschaften gehört in den Fachunterricht in den Schulen. Ein starkes Differenzempfinden resultiert zumeist aus dem Fehlen dieses Grundwissens.

- Hingegen kann die Verortung dieser Inhalte im Mediendiskurs einer weiteren Problemwahrnehmung dienen, weil von Medien allgemein erwartet wird, Themen dann aufzugreifen, wenn es Probleme gibt.

- Rassismus- und diskriminierungskritische Ansätze gehören in den Bildungskanon, ebenso wie Wissen über gruppendynamische und psychologische Prozesse, um Verwerfungen als Produkt solcher Prozesse zu erkennen und nicht der jeweils markierten Gruppe zuzuweisen.

- Als Demokratie benötigen wir einen systematischen Lehrplan Medienbildung, der (mediale) Meinungsbildungsprozesse kritisch reflektiert, sowie evaluiertes didaktisches Material. Die aktuellen Technisierungsinitiativen an Schulen gehen am Ziel vorbei.

- In Bezug auf die Berichterstattung über angeblich oder tatsächlich islamische Fragen sind die gleichen Standards anzuwenden, wie anderswo auch. ${ }^{29}$ Eine übermäßige Markierung von Muslimen führt zu einer stereotypen Zuweisung allgemein relevanter Fragestellungen auf diese Gruppe - etwa die Genderfrage. Die konsequente Anwendung von Qualitätsstandards (wozu auch Framing-Analysen gehören) gelten für alle Themen und sind immer wieder selbstkritisch zu prüfen.

- Eine „kultursensitive Berichterstattung“, die suggeriert, dass man Muslime in Watte packen müsse, operiert hingegen mit falschen Prämissen. Statt sog. Islamaufklärung (im falschen Kontext) wäre oftmals die Dekonstruktion der Zuweisungsprozesse Aufgabe der Medien - was übrigens auch auf andere Themen zutrifft, beispielsweise bezüglich des „Ossi“-Bilds.

- Die Anwendung journalistischer Standards müsste zu einer sorgfältigen Kontextklärung führen, die verhindert, dass Phänomene vorschnell oder monokausal „dem Islam“ zugewiesen werden. Schließlich lassen sich die

\footnotetext{
${ }^{29}$ Vgl. Pressekodex; http://www.presserat.de.
} 
historisch gewachsenen und global verstrickten Situationen in Ländern wie Tschetschenien, Pakistan oder Mali nicht reduktionistisch verstehen.

- Statt die gelungenen Beispiele von Inklusion und Erfolg besonders herauszuheben, was sie wiederum als Ausnahme markiert, ist längst Normalität angesagt. Und die ist sehr vielfältig, und dies kann gerade in den Unterhaltungsformaten gut dargestellt werden. ${ }^{30}$ Diversity Mainstreaming bedeutet eben gerade nicht eine besondere Thematisierung von Differenz, sondern das unmarkierte und damit gleichwertige Vorkommenlassen verschiedenster Menschen, Rollen und Lebensformen. ${ }^{31}$

\section{Self-fulfilling prophecy oder Durchbrechung des Teufelskreises?}

Das Potential, das unsere Medien haben, ist noch lange nicht ausgeschöpft. $\mathrm{Zu}$ glauben, dass sich alles schon richten werde, wenn der Islam als Religion in Deutschland anerkannt sei und die Muslime eine Körperschaft öffentlichen Rechts bildeten, entpuppt sich mit Blick auf die Situation in Österreich als naiv. ${ }^{32}$ Gerade die Missdeutungen von Diversity Mainstreaming und das Nichtausschöpfen der Potentiale im Unterhaltungsbereich, machen eine dem Nachrichtenwertfaktor Negativismus verpflichtete Berichterstattung zum Sprengsatz für gesellschaftlichen Zusammenhalt. Würden in den Unterhaltungsformaten kopftuchtragende Ärztinnen, dunkelhäutige Lehrkräfte und Kippa tragende Anwälte vorkommen, wäre auch ein türkischstämmiger Bankräuber leichter in die vorhandene Vielfalt einzuordnen und würde weniger schnell als Prototyp einer ganzen Gruppe wahrgenommen.

Als die EUMC (inzwischen FRA) ihren Bericht „Muslime in der Europäischen Union - Diskriminierung und Islamophobie" 2006 herausgab, ${ }^{33}$ hieß es in der Zusammenfassung bezüglich „Medien“:

Die Medien sollten ihre Berichterstattung überprüfen, um sicherzustellen, dass sie über diese Themen korrekt und umfassend informieren. Die Medien werden ermutigt, Maßnahmen zur Einstellung und Ausbildung von Journalisten zu initiieren, die

\footnotetext{
${ }^{30}$ Vgl. Andreas Dörner 2000, S. 164-178.

${ }^{31}$ Vgl. Sabine Schiffer 2010b.

${ }^{32}$ Vgl. John Bunzl und Kai Hafez 2009.

${ }^{33}$ Vgl. unter: http://fra.europa.eu/fraWebsite/attachments/Perceptions_DE.pdf (31.08.2012).
} 
die innerhalb der EU herrschende Vielfalt widerspiegeln. Die Mitgliedsstaaten werden ermutigt, in Übereinstimmung mit Artikel 14 der Richtlinie über den elektronischen Geschäftsverkehr (2000/31/EG) die entsprechenden für Internetanbieter geltenden Gesetze zu verabschieden oder zu verschärfen, um eine Weiterverbreitung von illegalem rassistischem Material zu verhindern. ${ }^{34}$

Neuere Untersuchungen zeigen, dass zwar Bildungsprogramme für Journalisten implementiert wurden - wie beispielsweise bei der Deutschen Welle aber ein unaufgeregter Umgang mit dem Thema weiterhin ein Desiderat in Deutschland, der EU und darüber hinaus bleibt. ${ }^{35}$ Solange man vonseiten des öffentlich-rechtlichen Rundfunks die Problematik immer wieder den privaten Rundfunkanbietern zuschiebt (wie in der Deutschen Islamkonferenz zwischen 2006 und 2009) oder man allenfalls gemeinsam gegen Blogs im Internet polemisiert (wie dies in der Schockphase nach den Morden in Norwegen 2010 geschah), wird es nur spärliche Fortschritte in Richtung Differenzierung und Deeskalation geben. Denn es liegt nicht am einzelnen Anderen, das Bild über die eigene Gruppe zu korrigieren, wie man aus dem antisemitischen Diskurs längst hätte lernen können.

Die Bevorzugung polarisierender Stimmen auch in den Mainstream-Medien befördert eher spaltende Tendenzen und nicht den gesellschaftlichen Zusammenhalt. Insofern sind heutige Phänomene von Segregation und Misstrauen aus journalistischer Sicht auch mit Blick auf die jüngere Geschichte zu betrachten und wiederum nicht vorschnell auf „kulturelle Eigenheiten“ zu projizieren.

Der in Spanien tätige niederländische Kommunikationswissenschaftler Teun van Dijk bescheinigt den europäischen Mediendiskursen insgesamt eine Tendenz zur Idealisierung der eigenen Gruppe. Das Rezept für diese Selbstidealisierung sei einfach, so van Dijk: „Betone deine guten Eigenschaften und die schlechten des anderen!“36 Insofern tut der Beginn jeder Auseinandersetzung

\footnotetext{
34 http://fra.europa.eu/fraWebsite/attachments/EUMC-highlights-DE.pdfS. 5(31.08.2012).

${ }^{35}$ Z. B. Kai Hafez und Carola Richter 2007; Dirk Halm ${ }^{2}$ 2008; Kay Sokolowsky 2009; Susan Schenk 2009; OECD Konferenz zum Thema Islamophobie (mit einer Sektion zu Medienfragen) am 28.10.2011 in Wien; Institute of Strategic Dialogue „Muslims in the European Mediascape“, unter: http://www. strategicdialogue.org/documents/Muslims\%20in\%20the\%20European\%20Mediascape\%20discussion\%20paper.pdf (31.08.2012). Präsentation vorläufiger Ergebnisse am 30.5.2012 unter: http://www.vodafone- stiftung.de/ presseinfomodul/detail/172.html (31.08.2012).

${ }^{36}$ Van Dijk 2007, S. 108.
} 
mit dem eigenen Splitter oder Balken im Auge gut, auch wenn man Vergleichbares im Auge des jeweiligen Gegenübers finden kann.

\section{Literatur}

Attia, Iman (Hrsg.), Orient- und Islambilder: Interdisziplinäre Beiträge zu Orientalismus und antimuslimischen Rassismus. Münster 2007.

Benz, Wolfgang (Hrsg.), Islamfeindschaft und ihr Kontext. Berlin 2009.

Benz, Wolfgang, Antisemitismusforschung als akademisches Fach und öffentliche Aufgabe, in: TUB-newsportal 16.11.2010.

Butterwegge, Christoph und Hentges Gudrun (Hrsg.), Massenmedien, Migration, Integration. Wiesbaden 2006.

Bunzl, John und Hafez, Farid, Islamophobie in Österreich. Wien 2009.

Dijk, Teun van, Rassismus und die Medien in Spanien, in: Jäger, Siegfried und Halm, Dirk (Hrsg.), Mediale Barrieren. Rassismus als Integrationshindernis. Münster 2007.

Dörner, Andreas, Das Kino als politische Integrationsagentur. Afro-amerikanische Identitätsangebote im Hollywood-Film der 90er Jahre, in: Schatz, Heribert u. a. (Hrsg.), Migranten und Medien. Neue Herausforderungen an die Integrationsfunktion von Presse und Rundfunk. Wiesbaden 2000.

Frindte, Wolfgang und Haußecker, Nicole (Hrsg.), Inszenierter Terrorismus. Mediale Konstruktionen und individuelle Interpretationen. Wiesbaden 2010.

Gleißner, Xenia und Schiffer, Sabine, Das Bild des Propheten, in: Paul, Gerhard (Hrsg.), Das Jahrhundert der Bilder, Bd. II - 1949 bis heute. Göttingen 2008, S. 750 ff.

Hafez, Kai, Die politische Dimension der Nahostberichterstattung. Bd. 2, München 2002.

Halm, Dirk, Der Islam als Diskursfeld. Bilder des Islams in Deutschland, 2. Auflage, Wiesbaden 2008 .

Heitmeyer, Wilhelm (Hrsg.), Deutsche Zustände. Folge 9/Dez. 2010.

Königseder, Angelika, Feindbild Islam, in: Benz, Wolfgang (Hrsg.), Islamfeindschaft und ihr Kontext. Berlin 2009.

Lakoff, George, Metaphor and War, in: alternet.org, 17.03.2003.

Link, Jürgen, Zur Archäologie der Bombenköpfe, in: Jäger, Siegfried und Halm, Dirk (Hrsg.), Mediale Barrieren. Rassismus als Integrationshindernis, Münster 2007, S. 151-165.

Rahman, Anika und Nahid Toubia, Female genital mutilation: a guide to laws and policies, in: "worldwide G - Reference, Information and Interdisciplinary Subjects Series". 2000, S. 209.

Rohe, Mathias, Der Islam - Alltagskonflikte und Lösungen. Rechtliche Perspektiven. 2. Auflage Freiburg 2001.

Said, Edward, Covering Islam, How the Media and the Experts Determine How We See the Rest of the World. Vintage Books, 2. Auflage 1997. 
Schenk, Susan, Das Islambild im internationalen Fernsehen: Ein Vergleich der Nachrichtensender Al Jazeera English, BBC World und CNN International. 2009.

Scheufele, Bertram, Frames - Framing - Framing-Effekte: Theoretische und methodische Grundlegung des Framing-Ansatzes sowie empirische Befunde zur Nachrichtenproduktion. Wiesbaden 2003.

Schiffer, Sabine, Die Darstellung des Islams in der Presse. Sprache, Bilder, Suggestionen. Würzburg 2005.

Schiffer, Sabine, Die Grenzen wohlmeinender Diskurse, in: migazin.de, 1.10.2010 (a).

Schiffer, Sabine, The Fiction of a Homogeneous National Culture, in: Anna Lindh Report 2010 (b), S. $48 \mathrm{f}$.

Schiffer, Sabine, Grenzenloser Hass im Internet. Wie „islamkritische“ Aktivisten in Weblogs argumentieren, in: Schneiders, Thorsten (Hrsg.), Islamfeindlichkeit. Wenn die Grenzen der Kritik verschwimmen. 2. Auflage Wiesbaden 2010 (c), S. 355-376.

Schiffer, Sabine, Medienverantwortung in Zeiten der Panikmache, in: migazin.de, 19.06.2012.

Schiffer, Sabine und Wagner, Constantin, Antisemitismus und Islamophobie - ein Vergleich. Wassertrüdingen 2009.

Shooman, Yasemin, Keine Frage des Glaubens. Zur Rassifizierung von „Kultur“ und „Religion“ im antimuslimischen Rassismus, in: Friedrich, Sebastian (Hrsg.), Rassismus in der Leistungsgesellschaft. Analysen und kritische Perspektiven zu den rassistischen Normalisierungsprozessen der ,Sarrazindebatte‘. Münster 2011.

Sokolowsky, Kay, Feindbild Moslem. Berlin 2009.

Spenlen, Klaus und Kröhnert-Othman, Susanne (Hrsg.), Integrationsmedium Schulbuch. Anforderungen an islamischen Religionsunterricht und seine Bildungsmaterialien, in: Eckert. Die Schriftenreihe, Band 132, Göttingen 2012.

\section{Internetquellen}

Hafez, Kai und Richter, Carola, Das Islambild von ARD und ZDF, in: bpb.de 21.06.2007 unter: www.bpb.de/publikationen/BSF019,0,Das_Islambild_von_ARD_und_ ZDF.html (31.08.2012). 
